\title{
FRATURAS DA DIÁFISE DO ÚMERO
}

\author{
HUMERAL SHAFT FRACTURES
}

Eduardo Benegas ${ }^{1}$, Arnaldo Amado Ferreira Neto ${ }^{2}$, Raul Bolliger Neto ${ }^{3}$, Flavia de Santis Prada ${ }^{1}$, Eduardo Angeli Malavolta', Gustavo Oliveira Marchitto ${ }^{4}$

\section{RESUMO}

As fraturas da diáfise do úmero (FDU) representam 3\% das fraturas do aparelho locomotor; o terço médio da diáfise direita é o mais acometido. Seu tratamento é, na sua maioria, realizado por meio de métodos não cirúrgicos, mas as indicações cirúrgicas nas FDU são adotadas em situações cada vez mais frequentes. A diversidade de opiniões torna difícil o consenso sobre qual o tipo de osteossíntese, qual a técnica cirúrgica, a quantidade e a qualidade dos materiais de síntese a serem utilizados. Temos a impressão de que o melhor método para o tratamento cirúrgico das FDU está longe de ter um consenso entre os especialistas. Acreditamos que os métodos menos invasivos e que privilegiam a estabilidade relativa são os mais adequados, pois complicações mais temidas são menos frequentes.

Descritores - Fraturas do úmero; Classificação; Tratamento; Cirurgia ortopédica; Fixação interna de fraturas

\section{ABSTRACT}

Humeral shaft fractures (HSF) represent 3\% of fractures of the locomotor apparatus, the mid-third section of the shaft being the most commonly affected. In the majority of cases, it is treated by non-surgical methods, but surgical indications in HSF are increasingly being adopted. The diversity of opinions makes it difficult to reach a consensus regarding to the type of osteosynthesis, surgical technique, and quantity and quality of the synthesis materials to be used. It would appear that specialists are far from reaching a consensus as to the best method for the surgical treatment of HSF. We believe that less invasive methods, which favor relative stability, are the most appropriate methods, as the most feared complications are less frequent.

Keywords - Humeral fractures; Classification; Treatment; Orthopedic surgery; Fractures fixation, internal

\section{INTRODUÇÃO}

As fraturas da diáfise do úmero (FDU) representam $3 \%$ das fraturas do aparelho locomotor ${ }^{(1)}$. Estima-se que em torno de 60 novos casos de FDU em adultos são tratados por ano para cada grupo de 600.000 habitantes $^{(2)}$.

Com relação à localização, o terço médio da diáfise direita é o mais acometido e o tipo A da classificação AO é o mais frequente $e^{(3)}$, sendo as do tipo $\mathrm{C}$ as mais raras ${ }^{(4,5)}$.

As fraturas expostas da diáfise do úmero são $\operatorname{raras}^{(2,6)}$.

O tratamento das FDU é, na sua maioria, realizado por meio de métodos não cirúrgicos, com bons resultados funcionais ${ }^{(4,7,8)}$. Entretanto, existem situações e determinados tipos de FDU para as quais o tratamento conservador não se mostra eficaz. Amparados pelos sensíveis progressos das últimas décadas na cirurgia do traumatismo do aparelho locomotor, com melhores técnicas e materiais de osteossíntese, as indicações cirúrgicas nas FDU são adotadas em situações cada vez mais frequentes, como traumatismos múltiplos, fraturas expostas, fraturas bilaterais, fraturas patológicas, etc ${ }^{(9)}$.

No tratamento cirúrgico das FDU, a técnica cirúrgica, a quantidade e a qualidade dos materiais de síntese utilizados ainda são motivos de controvérsias, que serão abordadas nesta revisão.

\footnotetext{
1 - Médico Assistente do Grupo de Ombro e Cotovelo do Instituto de Ortopedia e Traumatologia do HC/FMUSP, São Paulo, Brasil.

2 - Chefe do Grupo de Ombro e Cotovelo do Instituto de Ortopedia e Traumatologia do HC/FMUSP, São Paulo, Brasil.

3 - Médico Assistente do Instituto de Ortopedia e Traumatologia do HC/FMUSP, São Paulo, Brasil.

4 - Médico Assistente Colaborador do Grupo de Ombro e Cotovelo do Instituto de Ortopedia e Traumatologia do HC/FMUSP, São Paulo, Brasil.

Correspondência: Rua Ovídio Pires de Campos, 333, 3. ${ }^{\circ}$ and. - E-mail: e.benegas@terra.com.br
} 


\section{CLASSIFICAÇÃO}

Podemos classificar essas fraturas conforme o tipo do traço da fratura, a sua localização, se exposta ou fechada e conforme a condição do osso (normal ou patológico).

Uma das classificações mais utilizadas é a de Zuckerman e Koval ${ }^{(9)}$, que analisam a biomecânica dos desvios das FDU e afirmam que, quando o traço de fratura ocorre acima da inserção do músculo peitoral maior, o fragmento proximal estará desviado em abdução e rotação externa, por ação dos músculos do manguito rotador. Quando o foco de fratura está entre as inserções dos músculos peitoral maior e deltoide, o fragmento proximal estará em adução, por tração do músculo peitoral maior e o fragmento distal estará com desvio proximal e lateral, por tração do músculo deltoide. Quando o foco de fratura é distal à inserção do músculo deltoide, o fragmento proximal estará abduzido por tração do músculo deltoide e o distal estará com desvio proximal.

Mais completa é a classificação de Müller et $a l^{(3)}$, do grupo por eles fundado, denominado grupo $\mathrm{AO}$, onde as FDU são divididas conforme a Figura 1.

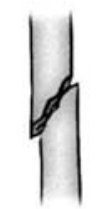

A

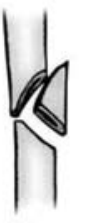

B

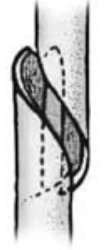

A 1

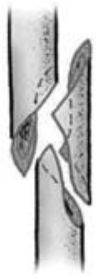

B 1
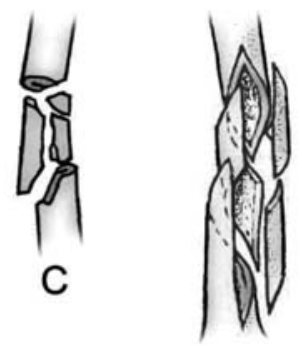

C 1

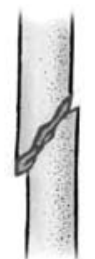

A 2

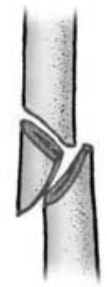

B 2

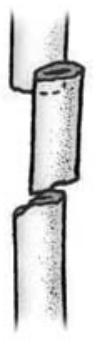

C 2

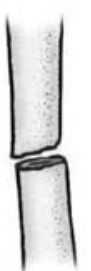

A 3

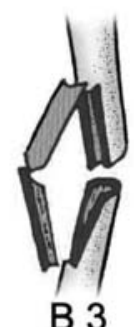

B 3
Figura 1 - Classificação AO das FDU

\section{DIAGNÓSTICO}

O exame radiográfico nas incidências de frente e perfil é suficiente para o diagnóstico e classificação das FDU.

A cintilografia óssea, ressonância magnética e tomografia computadorizada são utilizadas em situações especiais, como no diagnóstico e estadiamento das fraturas patológicas.

A eletroneuromiografia só será útil para o diagnóstico da lesão neurológica a partir da terceira semana do trauma; portanto, é imprescindível a realização do exame clínico adequado.

\section{TRATAMENTO}

Por ser o úmero um osso bem vascularizado e envolvido por vários músculos, o processo de consolidação é facilitado e também permite desvios (angulação anterior maior do que $20^{\circ}$, desvio em varo maior do que $30^{\circ}$, encurtamento maior do que três centímetros $^{(4,10)}$ ) sem alteração da função ou estética do membro superior.

Tais características explicam a opinião quase unânime de que o tratamento não cirúrgico conduz a altos índices de consolidação e bons resultados funcionais ${ }^{(4,5,7-9,11-14)}$.

Entre os diversos métodos de tratamento não cirúrgico (pinça de confeiteiro, gesso pendente, gesso toracobraquial e imobilização de Velpeau), o uso de órteses braquiais, que permitem a contração dos grupos musculares adjacentes e estimulam a consolidação, é o método de tratamento não cirúrgico mais utilizado atualmente ${ }^{(5,8)}$. Entretanto, existem determinados aspectos das FDU e do paciente que tornam difícil a condução do tratamento com imobilizações externas. Com o aumento das FDU associadas a politraumatismos, fraturas expostas, desvios causados por ação muscular ${ }^{(9)}$, além de outros fatores, tais como a obesidade, que levam a maus resultados com o tratamento não cirúrgico, muitos autores vêm procurando novos métodos de tratamento, como a utilização de $\operatorname{pinos}^{(15)}$, hastes intramedulares ${ }^{(16)}$ ou placas parafusadas ${ }^{(17,18)}$. Dessa forma, embora a maioria das FDU possa ser tratada de forma não cirúrgica, as características da fratura e as necessidades do paciente devem ser fundamentais na indicação operatória ${ }^{(14)}$.

Baseados nesta necessidade, Zuckerman e Koval ${ }^{(9)}$ indicam o tratamento cirúrgico nos casos de fratura exposta, lesão vascular associada, cotovelo flutuante, 
fratura segmentar, fratura patológica, fratura bilateral do úmero, fratura do úmero em pacientes politraumatizados, lesão do nervo radial após manipulação fechada das FDU, lesão nervosa após ferimento penetrante, FDU com desalinhamento inaceitável ou com extensão do traço de fratura para as articulações. Modabber e Jupiter ${ }^{(13)}$ utilizam o procedimento cirúrgico apenas nos casos de perda ou impossibilidade de redução da fratura, envolvimento das articulações no traço de fratura com desvio, fraturas associadas a lesões vasculares ou nervosas, outras fraturas no mesmo membro, fraturas segmentares, fraturas patológicas, fraturas expostas, pseudartroses, politraumatismos, fraturas bilaterais do úmero ou lesões do tegumento que impeçam o tratamento conservador. Rommens et $a l^{(18)}$ definem como indicações absolutas para a osteossíntese os politraumatismos, as fraturas expostas, as fraturas bilaterais do úmero, as fraturas patológicas, o cotovelo flutuante, as lesões vasculares e nervosas associadas, a paralisia do nervo radial após redução fechada e a pseudartrose e, como indicações relativas, as fraturas espirais longas, as fraturas transversas, as lesões do plexo braquial associadas, as paralisias nervosas primárias, a incapacidade de manter a redução, as doenças neurológicas, a falta de cooperação por abuso do álcool ou drogas e a obesidade.

Uma vez indicado o tratamento cirúrgico, a diversidade de opiniões torna difícil o consenso sobre qual o tipo de osteossíntese a ser utilizado.

Aqueles que defendem o tratamento com as placas e parafusos de $4,5 \mathrm{~mm}$ por via aberta ${ }^{(14,19-23)}$ acreditam que esse método leva a menor índice de complicações, como a lesão nervosa iatrogênica, pseudartrose, síndrome do impacto, fraturas durante a introdução das hastes por via retrógrada, capsulite adesiva, e a melhor resultado funcional, com menor tempo de imobilização pós-operatória, retorno mais rápido das funções articulares do ombro e cotovelo e melhor alinhamento da diáfise do úmero.

A experiência adquirida na traumatologia do IOTHCFMUSP nos faz acreditar que o envelope muscular e a vascularização em torno do foco da fratura devam ser preservados e que, quanto menor a dissecção das partes moles, menor o índice de complicações como infecção, lesão nervosa e pseudartrose.

De forma semelhante ao que pensam Ingman e Waters $^{(17)}$ e Habernek e Orthner ${ }^{(24)}$, preferimos as hastes bloqueadas intramedulares aos pinos intrame- dulares, pois aquelas garantem melhor fixação, o que permite mobilização precoce das articulações do ombro e cotovelo.

Também concordamos com Robinson et al ${ }^{(25)}$, que relatam que a fixação distal da haste desenvolvida por Seidel $^{(16)}$ é insuficiente e preferimos as hastes bloqueadas com parafusos proximais e um distal.

Contrários a Lin et al ${ }^{(26)}$, Ingman e Waters ${ }^{(17)} \mathrm{e}$, da mesma forma que Habernek e Orthner ${ }^{(24)}$, Modabber e Jupiter $^{(13)}$, Flinkkilä et $a l^{(27)}$, acreditamos que a introdução anterógrada da haste intramedular bloqueada, com dissecção cuidadosa do manguito rotador e profundamente à cortical proximal da tubérculo maior, minimiza os riscos de dores residuais no ombro.

Concordamos com Modabber e Jupiter ${ }^{(13)}$, que quando utilizam as hastes intramedulares preferem não fresar o canal medular, pois assim danificam menos a circulação do endósteo. Também concordamos com o uso de hastes não fresadas, já que o úmero, por ser um osso do membro superior, não sofre carga axial, tornando desnecessário o uso de fresas para alargamento do canal medular. Outro argumento para o uso de hastes não fresadas foi defendido por Verbruggen et al ${ }^{(28)}$, que afirmam que tanto as hastes fresadas quanto as não fresadas são capazes de resistir às forças deformantes das FDU.

Embora acreditemos que a osteossíntese das FDU com fixador externo seja um bom método para o tratamento das fraturas expostas ou para o controle de danos nos politraumatizados, concordamos com Rommens et $a l^{(18)}$, em que o uso prolongado desse fixador no tratamento definitivo dessa fratura causa complicações como infecções e soltura dos parafusos de fixação.

Livani e Belangero ${ }^{(29,30)}$ publicam artigo científico com técnica original, onde tratam as FDU pela técnica da placa em ponte (PP), já consagrada para tratamento de outros ossos longos, mas, até então, não utilizada para o úmero por medo da lesão iatrogênica do nervo radial. Entusiasmados com os bons resultados publicados por Livani e Belangero ${ }^{(29,30)}$ e com as vantagens do método de estabilidade relativa, que não disseca o manguito rotador, que protege o envelope muscular e a vascularização em torno do foco da fratura e evita a lesão iatrogênica do nervo radial, planejamos um estudo prospectivo e randomizado comparando a haste intramedular bloqueada (HIB) com a placa em ponte para o tratamento cirúrgico das FDU (Figuras 2 e 3). 


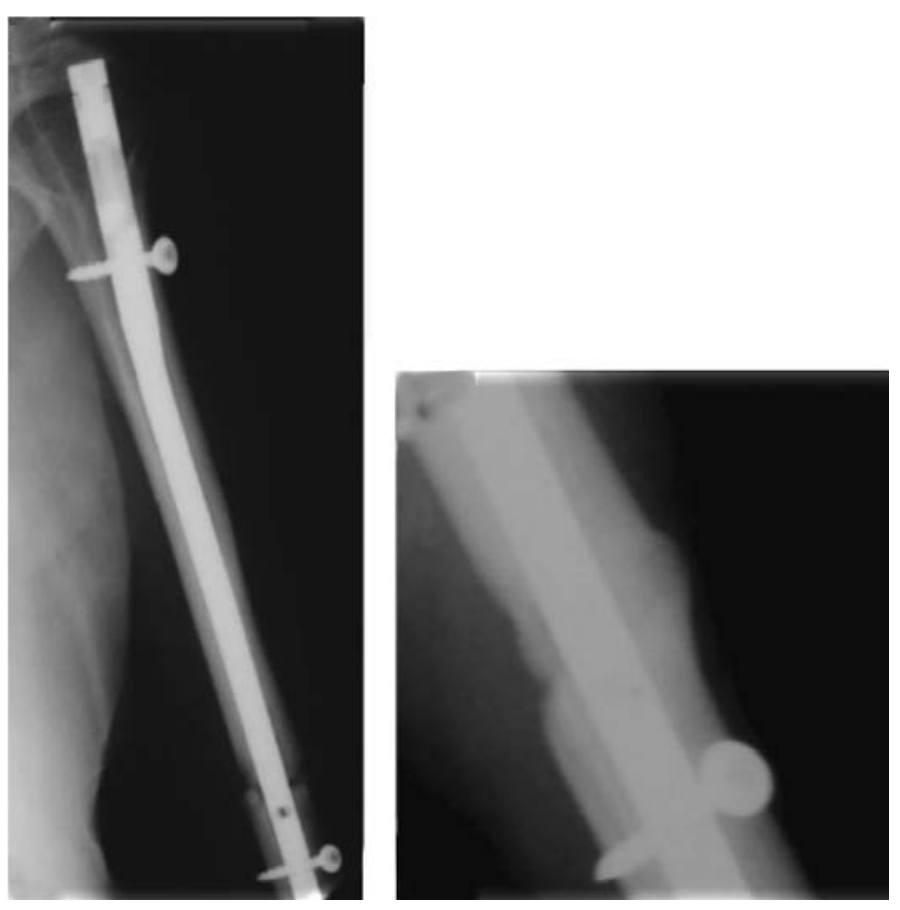

Figura 2 - Haste intramedular bloqueada
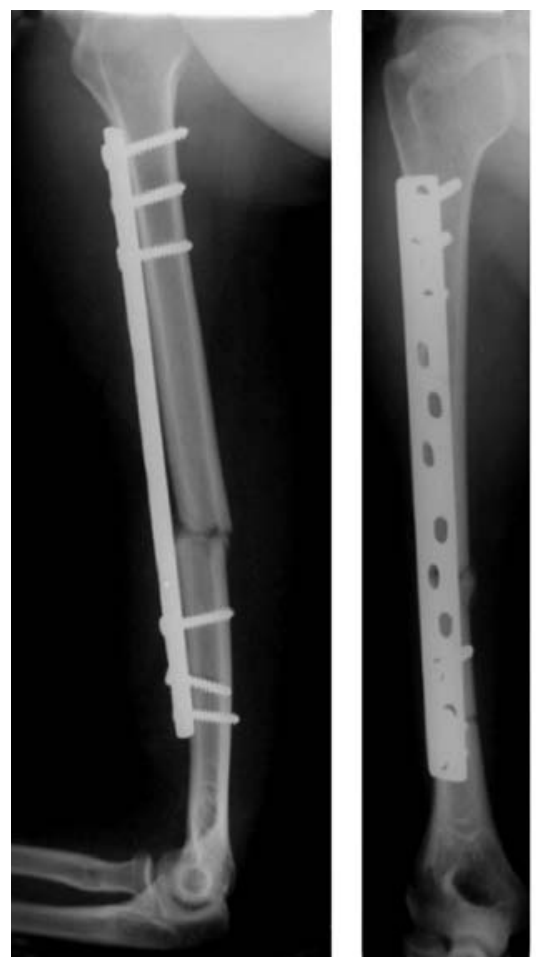

Figura 3 - Placa em ponte

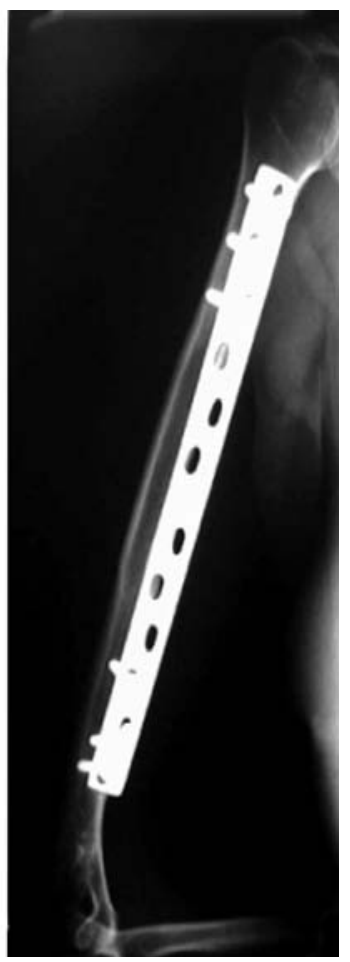

A consolidação ocorreu em 100\% do grupo PP e 94,7\% do grupo HIB, números bastante próximos aos encontrados por Sarmiento et $a l^{(8)}$, de $98 \%$, com o tratamento não cirúrgico das FDU. Outros índices de consolidação com o uso de hastes intramedulares são relacionados nos trabalhos de Ingman e Waters ${ }^{(17)}$ com 97,6\%, Rommens et al ${ }^{(18)}$ com 94,8\% e Scheerlinck e Handelberg ${ }^{(31)}$ com 93\%.

Comprovamos que, nas técnicas utilizadas, o tempo de utilização da radioscopia é maior quando empregamos HIB.

Embora tenhamos utilizado maior tempo de radioscopia no grupo HIB, o tempo cirúrgico não apresentou diferença estatística significante.

O resultado funcional final dos métodos PP ou HIB foram semelhantes aos obtidos por autores como Mast et $a l^{(7)}$ e Sarmiento ${ }^{(8)}$, com o tratamento não cirúrgico; Rommens et al $^{(18)}$ com a utilização de hastes intramedulares e Changulani et $a l^{(32)}$, com o uso da placa e parafusos por via aberta, ficando em torno de $85 \%$ de resultados satisfatórios.

Cumpre lembrar que, da mesma forma que para Gadegone e Salphale ${ }^{(33)}$, as FDU associadas com a lesão prévia do nervo radial não foram incluídas no nosso estudo para não influenciar no resultado final comparativo entre dois métodos de tratamento. Para Shao et $a^{(34)}$, essas lesões ocorrem em 11,8\% dos casos e regridem espontaneamente em 70,7\% das ocorrências, a partir da sétima semana, tempo idêntico ao encontrado por Ring et $a^{(35)}$. Pollock et $a^{(36)}$ encontram apenas 6\% de lesão do nervo radial associada às FDU, com 92\% de regressão espontânea dos sintomas, e recomendam aguardar de três meses e meio a quatro meses antes de uma exploração cirúrgica.

\section{CONCLUSÃO}

Ao final deste estudo ficou a impressão de que o melhor método para o tratamento cirúrgico das FDU está longe de ter um consenso entre os especialistas que se dedicam ao seu estudo, pois cada método tem suas vantagens, desvantagens e complicações próprias. Acreditamos que os métodos menos invasivos e que privilegiam a estabilidade relativa, como a HIB ou a PP, são os mais adequados, pois as complicações mais temidas que, em nossa opinião, são as infecções, lesões neurovasculares e as pseudartroses do úmero, são menos frequentes. Entre esses dois métodos de estabilidade relativa (HIB e PP), este último parece-nos o mais recomendável, pois, além de proporcionar altos índices de consolidação e resultados satisfatórios, expõe menos o paciente e o cirurgião aos raios $\mathrm{X}$ do que a técnica com HIB. 


\section{REFERÊNCIAS}

1. Christensen S. Humeral shaft fractures, operative and conservative treatment. Acta Chir Scand. 1967;133(6):455-60.

2. Tytherleigh-Strong G, Walls N, McQueen MM. The epidemiology of humeral shaft fractures. J Bone Joint Surg Br. 1998;80(2):249-53.

3. Müller ME, Allgöwer M, Schneider R, Willenegger $\mathrm{H}$. Manual of internal fixation. 4th ed. New York: Springler-Verlog; 1991. p.118-20.

4. Klenerman L. Fractures of the shaft of the humerus. J Bone Joint Surg Br. 1966;48(1):105-11.

5. Zagorski JB, Latta LL, Zych GA, Finnieston AR. Diaphyseal fractures of the humerus. Treatment with prefabricated braces. J Bone Joint Surg Am. 1988;70(4):607-10.

6. Benegas E, Amodio DT, Correia LFM, Malavolta EA, Ramadan LB, Ferreira Neto AA. et al. Estudo comparativo prospectivo e randomizado entre o tratamento cirúrgico das fraturas diafisárias do úmero com placa em ponte e haste intramedular bloqueada (análise preliminar). Acta Ortop Bras. 2007;15(2):87-92.

7. Mast JW, Spiegel PG, Harvey JP, Harrison C. Fractures of the humeral shaft. A retrospective study of 240 adult fractures. Clin Orthop Relat Res. 1975;(112):254-62.

8. Sarmiento A, Kinman PB, Galvin EG, Schmitt RH, Phillips JG. Functional bracing of fractures the shaft of the humerus. J Bone Joint Surg Am. 1977;59(3)596-601.

9. Zuckerman JD, Koval KJ. Fractures of the shaft of the humerus. In: Rockwood CA Jr, Green DP, editors. Fractures in adults. 4th ed. Philadelphia: Lippincott \& Raven; 1996. p.1025.

10. Klenerman L. Injuries of arm. In: Jones W. Fracture and joint injuries. 6th ed. Edinburgh: Churchill Livingstone; 1982.

11. Charnley J. The closed treatment of common fractures. 3rd ed. London: ES Livingston; 1968.

12. Souza RS, Marcio MP, Gottfried K, Davitt M. Tratamento functional das fraturas diafisárias do úmero com caneleiras de futebolista. Rev Bras Ortop. 1985;20(3):114-8.

13. Modabber MR, Jupiter JB. Operative management of diaphyseal fractures of the humerus. Plate versus nail. Clin Orthop Relat Res. 1998;(347):93-104.

14. Schemitsh EH, Bhandari M. Fractures of the humeral shaft. In: Browner B, Jupiter J, Levine A, Trafton P. Skeletal Trauma. 3th ed. Philadelphia: Saunders; 2003. p.1481-511.

15. Rush LV, Rush HL. Intramedullary fixation of fractures of the humerus by the longitudinal pin. Surgery. 1950;27(2):268-75.

16. Seidel H. Humeral locking nail: a preliminary report. Orthopedics. 1989; 12(2):219-26.

17. Ingman AM, Waters DA. Locked intramedullary nailing of humeral shaft fractures. Implant desing technique and clinical results. J Bone Joint Surg Br. 1994;76(1):23-9.

18. Rommens PM, Verbruggen J, Broos PL. Retrograde locked nailing of humeral shaft fractures. J Bone Joint Surg Br. 1995;77(1):84-9.

19. Holstein A, Lewis GB. Fractures of the humerus with radial-nerve paralysis. J Bone Joint Surg Am. 1963;45(10):1382-8.

20. McCormack RG, Brien D, Buckley RE, McKee MD, Powell J, Schemitsch EH.
Fixation of fractures of the shaft of the humerus by dynamic compression plate or intramedullary nail. A prospective, randomised trial. J Bone Joint Surg Br. 2000;82(3):336-9.

21. Kesemenli CC, Subaşi M, Arslan H, Necmioğlu S, Kapukaya A. Comparison between the results of intramedullary nailing and compression plate fixation in the treatment of humerus fractures. Acta Orthop Traumatol Turc. 2003;37(2):120-5.

22. Bhandari M, Devereaux PJ, McKee MD, Schemitsch EH. Compression plating versus intramedullary nailing of humeral shaft fractures: a meta-analysis. Acta Orthop. 2006;77(2):279-84.

23. Jawa A, McCarty P, Doornberg J, Harris M, Ring D. Extra-articular distal-third diaphyseal fractures of the humerus. J Bone Joint Surg Am. 2006;88(11):2343-7.

24. Habernek $\mathrm{H}$, Orthner E. A locking nail for fractures of the humerus. J Bone Joint Surg Br.1991;73(4):651-3.

25. Robinson CM, Bell KM, Court-Brown CM, McQueen MM. Locked nailing of humeral shaft fractures. Experience in Edinburgh over a two-year period. J Bone Joint Surg Br. 1992;74(4):558-62.

26. Lin J, Shen PW, Sheng-Mon H. Complications of locked nailing in humeral shaft fractures. J Trauma. 2002;54(4):943-9.

27. Flinkkilä $T$, Hyvönen $P$, Siira $P$, Hämäläinen $M$. Recovery of shoulder joint function after humeral shaft fracture: a comparative study between antegrade intramedullary nailing and plate fixation. Arch Orthop Trauma Surg. 2004;124(8):537-41.

28. Verbruggen JP, Sternstein W, Blum J, Rommens PM, Stapert JW. Compression-locked nailing of the humerus: a mechanical analysis. Acta Orthop. 2007;78(1):143-50

29. Livani B, Belangero BD. Bridging plate osteosynthesis of humeral shaft fractures. Injury. 2004;35(6):587-95.

30. Livani B, Belangero WD. Osteossíntese de fratura diafisária do úmero com placa em ponte: apresentação e descrição da técnica. Acta Ortop Bras. 2004;12(2):113-7.

31. Scheerlinck T, Handelberg F. Functional outcome after intramedullary nailing of humeral shaft fractures: comparison between retrograde Marchetti-Vicenzi and unreamed AO antegrade nailing. J Trauma. 2002;52(1):60-71.

32. Changulani M, Jain UK, Keswani T. Comparison of the use of the humerus intramedullary nail and dynamic compression plate for the management of diaphyseal fractures of the humerus. A randomised controlled study. Int Orthop. 2007;31(3):391-5.

33. Gadegone WG, Salphale Y. Antegrade Rush nailing for fractures of humeral shaft: an analysis of 200 cases with an average follow-up of 1 year. Indian J Orthop. 2006;40(1):180-2.

34. Shao YC, Harwood P, Grotz MR, Limb D, Giannoudis PV. Radial nerve palsy associated with fractures of the shaft of the humerus: a systematic review. J Bone Joint Surg Br. 2005;87(12):1647-52.

35. Ring D, Chin K, Jupiter JB. Radial nerve palsy associated with high-energy humeral shaft fractures. J Hand Surg Am. 2004;29(1):144-7.

36. Pollock FH, Drake D, Bovill EG, Day L, Trafton PG. Treatment of radial neuropathy associated with fractures of the humerus. J Bone Joint Surg Am. 1981;63(2):239-43 